\title{
On a cotangent form of a nonholonomic lagrangian dynamics
}

\author{
Paul Popescu ${ }^{1, *}$ and Marcela Popescu ${ }^{1, * *}$ \\ ${ }^{1}$ Department of Applied Mathematics \\ University of Craiova \\ 200585 Craiova, Romania
}

\begin{abstract}
A Lagrangian form of dynamic equations for nonlinear nonholonomic constraints was studied by the first author in a previous paper. The aim of this paper is to put these equations in a cotangent form, according to some regularity conditions. It is particularized as an example to a decelerated motion of a free particle, when some dual simple equations are obtained.
\end{abstract}

\section{Introduction}

The geometrization of nonholonomic systems is a historically outstanding problem in mechanics and geometry (see, for example [14]). In the study of nonholonomic systems one give constraints of motion; most used and studied ones are those linear and affine in velocities. See, for example, monograph [6] and the citations therein. But nonlinear constraints are also involved in nonholonomic mechanics; see, for example [20] and the citations therein.

The Chetaev principle, generally accepted in nonlinear constraint case, comes from the following principle: take the variation before imposing the constraints, that is, not imposing the constraints on the family of curves defining the variation. In this case we follow similar arguments as in the linear and affine constraints in the above papers of A. M. Bloch and we given a new form expressed in Theorem 3.1. Adapting these results in the case of time dependent nonlinear constraints, we obtain a similar general result that applies in the cases of generalized Chetaev case [16] or the examples therein.

Some criticism concerning Chetaev's principle is drawn, for example, in [17, 18], where some situations (as Appell machine) are presented as examples when Chetaev principle fails to a real situation. Our goal was not to study the workability of Chetaev or generalized Chetaev principle, but the possibility to put in an unitary form the dynamics equations coming from linear, affine and regular nonlinear constraints.

The setting uses foliations. Nonlinear constraints, including linear and affine ones, are considered for lagrangians, but following the classical bundle setting as used by A. M. Bloch for linear and affine constraints [6]. The implicit forms of constraints and a link with the Lagrange multipliers form of Euler-Lagrange equation can be considered.

Given a nonlinear constraint $C$, then an almost transverse semi-spray gives a $C$-semispray $S$ (Proposition 3.3) and a $C$-semispray $S$ gives rise to an $S$-curvature $R$ of $C$ (Proposition

\footnotetext{
*e-mail: paul_p_popescu@yahoo.com

**e-mail: marcelacpopescu@yahoo.com
} 
3.4). An $S$-curvature $R$ of $C$ is considered, in the case of nonlinear constraints, extending the cases of linear and affine constraints, when the curvature $R$ of $C$ appears more simply defined.

The result stated in Theorem 3.1 gives a synthetic form of linear and regular-nonlinear cases. This result can be adapted to other situations; for example, in the case of time dependent constraints, or of a time independent lagrangian (as studied in [16] and the examples of the Appell-Hammel dynamic system in an elevator and the riemannian flow (see [20]).

In order to illustrate the constructions performed in the lagrangian case, some examples are considered here: the linear and nonlinear Appell machine and a riemannian flow, which is particularized to a decelerated motion of a free particle, as studied in $[12,22]$.

The main result of the paper is Theorem 4.1, where an alternative cotangent form to the lagrangian form from Theorem 3.1 is given. For a decelerated motion of a free particle we give both tangent and cotangent forms of its dynamic equations.

We notice that the lagrangian setting is based on the source [20].

We use basic constructions and results on vector bundles and foliations from [7, 19].

We notice also that almost all formulas obtained, except for some explicit situations, have the same form in the simple (i.e. fibered manifold) case, as well as in the foliated case.

\section{Preliminaries on foliations}

Let us consider an $(n+m)$-dimensional manifold $M$, connected and orientable. A codimension $n$ foliation $\mathcal{F}$ on $M$ is defined by a foliated cocycle $\left\{U_{i}, \varphi_{i}, f_{i, j}\right\}$.

Every fibre of $\varphi_{i}$ is called a plaque of the foliation. The manifold $M$ is decomposed into submanifolds, called leafs of $\mathcal{F}$. If $U \subset M$ is an open subset, then there is an inducedfoliation $\mathcal{F}_{U}$.

We denote by $T \mathcal{F}$ the tangent bundle to $\mathcal{F}$ and by $\Gamma(T \mathcal{F})$ the module of its global sections, i.e. the vector fields on $M$ tangent to $\mathcal{F}$. The normal bundle of $\mathcal{F}$ is $N \mathcal{F}=T M / T \mathcal{F}$. A vector field on $M$ is transverse if it locally projects to the transversal manifold.

A system of local coordinates adapted to $\mathcal{F}$ are coordinates $\left(x^{u}, x^{\bar{u}}\right), u=1, \ldots, m, \bar{u}=$ $1, \ldots, n$ on an open subset $U$, where $\mathcal{F}_{U}$ is trivial and defined by the equations $d x^{\bar{u}}=0, \bar{u}=$ $1, \ldots, n$.

A particular example of a foliation is a fibered manifold, called a simple foliation. In particular, a locally trivial fibration.

There are elementary examples of simple foliations that come from no trivial fibrations and the spaces of leaves are not Hausdorff separated.

\section{Linear and affine constraints and Lagrangians}

The linear case is a simple transcription to foliations of the fiber bundle case (as for example Bloch's monograph).

A linear constraint system of a foliation $\mathcal{F}$ is a left splitting of the inclusion $T \mathcal{F} \stackrel{I_{0}}{\rightarrow} T M$. Since there is a short exact sequence of vector bundle morphisms

$$
0 \rightarrow T \mathcal{F} \stackrel{I_{0}}{\rightarrow} T M \stackrel{\Pi_{0}}{\rightarrow} N \mathcal{F} \rightarrow 0
$$

it follows that the existence of a left splitting $C$ of $I_{0}$ is equivalent to the existence of a right splitting $D$ of the projection $\Pi_{0}$, thus with an inclusion of $N \mathcal{F}$ in $T M$, via the injective morphism $D$, that gives a decomposition $T M=T \mathcal{F} \oplus H \mathcal{F}$, where $H \mathcal{F}=D(N \mathcal{F})$. The curvature of $C$ is the bilinear map $B: \Gamma(N \mathcal{F}) \times \Gamma(N \mathcal{F}) \rightarrow \Gamma(T \mathcal{F})$ given by

$$
B(X, Y)=C([D(X), D(Y)]) .
$$


The curvature has the form

$$
B(\bar{X}, \bar{Y})=C\left(\left[\bar{X}^{h}, \bar{Y}^{h}\right]\right) .
$$

Using local coordinates: $\left(x^{u}, x^{\bar{u}}, y^{u}, y^{\bar{u}}\right) \stackrel{C}{\rightarrow}\left(x^{u}, x^{\bar{u}}, y^{u}+C_{\bar{u}}^{u}\left(x^{u}, x^{\bar{u}}\right) y^{\bar{u}}\right)$ and the corresponding $D$ is $\left(x^{u}, x^{\bar{u}}, y^{\bar{u}}\right) \stackrel{D}{\rightarrow}\left(x^{u}, x^{\bar{u}},-C_{\bar{u}}^{u}\left(x^{u}, x^{\bar{u}}\right) y^{\bar{u}}, y^{\bar{u}}\right)$.

The curvature $B$ of $C$ has the local form

$$
\begin{aligned}
B_{\bar{u} \bar{v}}^{u} \frac{\partial}{\partial x^{u}} & =B\left(\frac{\delta}{\delta x^{\bar{u}}}, \frac{\delta}{\delta x^{\bar{v}}}\right)=\left[\frac{\delta}{\delta x^{\bar{u}}}, \frac{\delta}{\delta x^{\bar{v}}}\right] \\
& =\left(\frac{\partial C_{\bar{u}}^{u}}{\partial x^{\bar{v}}}-\frac{\partial C_{\bar{v}}^{u}}{\partial x^{\bar{u}}}+C_{\bar{v}}^{v} \frac{\partial C_{\bar{u}}^{u}}{\partial x^{v}}-C_{\bar{u}}^{v} \frac{\partial C_{\bar{v}}^{u}}{\partial x^{v}}\right) \frac{\partial}{\partial x^{u}},
\end{aligned}
$$

where

$$
\frac{\delta}{\delta x^{\bar{u}}}=\left(\frac{\partial}{\partial x^{\bar{u}}}\right)^{h}=\frac{\partial}{\partial x^{\bar{u}}}-C_{\bar{u}}^{u}\left(x^{u}, x^{\bar{u}}\right) \frac{\partial}{\partial x^{u}} .
$$

The manifold is $M=R^{3} \times T^{2}$ and the foliation is the simple foliation defined by the fibers of the canonical projection $R^{3} \times T^{2} \rightarrow T^{2}$. Consider the coordinates $\left(x^{1}, x^{2}, x^{3}\right)$ on $R^{3}$ and $\left(x^{\overline{1}}, x^{\overline{2}}\right)$ on $T^{2}$. The linear Appell constraints are given by the formulas

$$
C^{1}=R y^{\overline{1}} \cos x^{\overline{2}}, C^{2}=R y^{\overline{1}} \sin x^{\overline{2}}, C^{3}=r y^{\overline{1}} .
$$

Using formulas (4), its curvature $B$ has the coefficients

$$
B_{\overline{1} \overline{2}}^{1}=-R \sin x^{\overline{2}}, B_{\overline{1} \overline{2}}^{2}=R \cos x^{\overline{2}}, B_{\overline{1} \overline{2}}^{3}=0 .
$$

An affine constraint system of a foliation $\mathcal{F}$ is a fibered map $D^{\prime}: N \mathcal{F} \rightarrow T M$ affine on fibers. One can decompose $D^{\prime}$ as

$$
D^{\prime}(\bar{X})=D(\bar{X})-b
$$

where $D$ comes from a linear constraint $C: T M \rightarrow T \mathcal{F}$ and $b \in \Gamma(T \mathcal{F})$ is a tangent vector field to $\mathcal{F}$. We can also define a map $C^{\prime}: T M \rightarrow T \mathcal{F}$, by $C^{\prime}(X)=C(X)+b$. In the affine case, it can be easily seen that giving $C$ and $b$ is equivalent to giving $D$ and $b$.

In a similar way one can extend the definition of an adapted Lagrangian $L$, asking that $L$ has the form

$$
L(X)=L_{0}\left(C^{\prime}(X)\right)+\bar{L}\left(\Pi_{0}(X)\right), X \in X(\widetilde{T M}),
$$

where $C^{\prime}$ is an affine constraint and $\widetilde{T M}=T M-$ zzero section $\}$.

According to [6, Ch. 5], a covariant derivative of $b$, along a horizontal vector field $\bar{X} \in$ $\Gamma(D(N \mathcal{F}))$, can be considered as a vector field $\nabla_{\bar{X}} b \in \mathcal{X}(M)$ that projects by $\Pi_{0}$ on $\bar{X}$. Using local coordinates, if a linear constraint has the local form (3), then $C$ (corresponding to $D$ ) and $D^{\prime}$ have the forms (3) and

$$
\left(x^{u}, x^{\bar{u}}, y^{\bar{u}}\right) \stackrel{D^{\prime}}{\rightarrow}\left(x^{u}, x^{\bar{u}}, b^{u}\left(x^{u}, x^{\bar{u}}\right)-C_{\bar{u}}^{u}\left(x^{u}, x^{\bar{u}}\right) y^{\bar{u}}\right)
$$

respectively. If

$$
\bar{X}=\bar{X}^{u}\left(\frac{\partial}{\partial x^{\bar{u}}}-C_{\bar{u}}^{u} \frac{\partial}{\partial x^{u}}\right),
$$

then

$$
\left(x^{u}, x^{\bar{u}}\right) \stackrel{\nabla_{\bar{X}} b}{\rightarrow}\left(x^{u}, x^{\bar{u}}, \bar{X}^{\bar{v}} \gamma_{\bar{v}}^{u}, \bar{X}^{\bar{u}}\right),
$$


where

$$
\gamma_{\bar{u}}^{u}=\frac{\partial b^{u}}{\partial x^{\bar{u}}}-C_{\bar{u}}^{v} \frac{\partial b^{u}}{\partial x^{v}}+b^{v} \frac{\partial C_{\bar{u}}^{u}}{\partial x^{v}} .
$$

Using nonlinear constraints approach, studied in the next sections, the curvature $R$ of $D$ can be expressed also by the formula

$$
R_{\bar{u}}^{u} \frac{\partial}{\partial x^{u}}=-\left[\left[\frac{\partial}{\partial y^{\bar{u}}}, C_{V}\right], C_{V}\right]=\left(\gamma_{\bar{u}}^{u}+B_{\bar{u} \bar{v}}^{u} y^{\bar{v}}\right) \frac{\partial}{\partial x^{u}},
$$

where $C_{V}=\left(C_{\bar{u}}^{u} y^{\bar{u}}-b^{u}\right) \frac{\partial}{\partial x^{u}}+y^{\bar{u}} \frac{\partial}{\partial x^{\bar{u}}}$. According to Proposition 3.4 in the next section, the curvature is represented by a global tensor $R \in L\left(V N \mathcal{F}, T \mathcal{F}_{N \mathcal{F}}\right)$, while $C_{V}$ is not a tensor, having only a local vector field form. This curvature and the covariant derivative play an important role in [6, Sect. 5.2] to express the nonholonomic equations of motion, in the case of linear and affine constraints.

Let us consider the endomorphism $\tilde{J} \in \operatorname{End}(T N \mathcal{F})$, induced by the projection of the canonical almost tangent structure $J \in \operatorname{End}(T T M)$.

We say that a map $C: N \mathcal{F} \rightarrow T M$, viewed also as a section $C \in \Gamma\left(\pi_{N \mathcal{F}}^{*} T M\right)$, is a nonlinear constraint if $\tilde{J}(C)=\Gamma_{0}$. Using local coordinates, $\left(x^{u}, x^{\bar{u}}, y^{\bar{u}}\right) \stackrel{C}{\rightarrow}$ $\left(x^{u}, x^{\bar{u}}, C^{u}\left(x^{v}, x^{\bar{v}}, y^{\bar{v}}\right), y^{\bar{u}}\right), C=C^{u} \frac{\partial}{\partial x^{u}}+y^{\bar{u}} \frac{\partial}{\partial x^{\bar{u}}}$.

Proposition 3.1 [20, Proposition 1]A nonlinear constraint gives rise to a left splitting $C^{\prime \prime}$ or, equivalently, to a right splitting $D^{\prime \prime}$ of the exact sequence of vector bundle morphisms

$$
0 \rightarrow \pi_{N \mathcal{F}}^{*} T \mathcal{F} \stackrel{I_{0}^{\prime \prime}}{\rightarrow} \pi_{N \mathcal{F}}^{*} T M \stackrel{\Pi_{0}^{\prime \prime}}{\rightarrow} \pi_{N \mathcal{F}}^{*} N \mathcal{F} \rightarrow 0 .
$$

It follows that there is an inclusion of $\pi_{N \mathcal{F}}^{*} N \mathcal{F}$ in $\pi_{N \mathcal{F}}^{*} T M$, via the injective morphism $D^{\prime \prime}$, that gives a decomposition

$$
\pi_{N \mathcal{F}}^{*} T M=\pi_{N \mathcal{F}}^{*} T \mathcal{F} \oplus N^{\prime \prime} \mathcal{F}
$$

where $N^{\prime \prime} \mathcal{F}=D^{\prime \prime}\left(\pi_{N \mathcal{F}}^{*} N \mathcal{F}\right)$.

We say that $F$ is a contravariant implicit constraint (or a con-constraint for short) if

- for every $x \in M$ and any transverse vector $\bar{X}_{x} \in N_{x} \mathcal{F}$, there is a point in $T_{x} M \cap \mathcal{C}_{F}$ that projects on $\bar{X}_{x}$;

- the local matrices $\left(\frac{\partial F^{u}}{\partial y^{v}}(z)\right)$ are non-singular in all $z \in C_{F}$.

Analogously one define a covariant implicit constraint (or a cov-constraint for short).

The implicit form of constraints can be used to give an invariant form to the condition that a covector type be a combination of partial derivatives of functions that give the constraints; for example, in nonholonomic mechanics, the Chetaev condition reads that the covector giving the Euler-Lagrange derivative is such a combination.

Proposition 3.2 [20, Proposition 2]Let $E: T \mathcal{F} \rightarrow T^{*} M$ be a fibered manifold map over the base $M$. If $E=E_{u} d x^{u}+E_{\bar{u}} d x^{\bar{u}}$ has the property

$$
E_{u}=\sum_{v} \lambda_{v} \frac{\partial F^{v}}{\partial y^{u}}\left(x^{u}, x^{\bar{u}}, C^{u}, y^{\bar{u}}\right), E_{\bar{u}}=\sum_{v} \lambda_{v} \frac{\partial F^{v}}{\partial y^{\bar{u}}}\left(x^{u}, x^{\bar{u}}, C^{u}, y^{\bar{u}}\right)
$$

on $C_{F}$, for a given con-constraint $F$, or

$$
E_{u}=\sum_{v} \lambda^{v} \frac{\partial F_{v}}{\partial y^{u}}\left(x^{u}, x^{\bar{u}}, C^{u}, y^{\bar{u}}\right), E_{\bar{u}}=\sum_{v} \lambda^{v} \frac{\partial F_{v}}{\partial y^{\bar{u}}}\left(x^{u}, x^{\bar{u}}, C^{u}, y^{\bar{u}}\right)
$$


on $C_{F}$, for a given cov-constraint $F$, then the following identity holds true:

$$
E_{\bar{u}}+\sum_{u} \frac{\partial C^{u}}{\partial y^{\bar{u}}} E_{u}=0
$$

Nonlinear constraints lift to linear constraints of the natural lifted foliation $\mathcal{F}_{N \mathcal{F}}$ on $N \mathcal{F}$. The formulas $C_{\bar{u}}^{u}=\frac{\partial C^{u}}{\partial y^{u}}, C_{v}^{u}=0$ give rise to a linear constraint on $\mathcal{F}_{N \mathcal{F}}$, i.e. a splitting (left $C$ or right $\mathcal{D}$ ) of the exact sequence

$$
0 \rightarrow T \mathcal{F}_{N \mathcal{F}} \stackrel{I_{0}^{\prime}}{\rightarrow} T(N \mathcal{F}) \stackrel{\Pi_{0}^{\prime}}{\rightarrow} N \mathcal{F}_{N \mathcal{F}} \rightarrow 0
$$

An almost transverse semi-spray is a (non-necessarily foliated) section $S: N \mathcal{F} \rightarrow N N \mathcal{F}$ that is a section for both vector bundle structures on $N N \mathcal{F}$ over $N \mathcal{F}$ (one of usual vector bundle, the other one induced by the transversal component of the differential of the canonical projection $N \mathcal{F} \rightarrow M$, as a foliated map). In the case of the trivial foliation by the points of $M$, we recover the definition of a semi-spray on $M$.

Proposition 3.3 [20, Proposition 3]If $S \in \Gamma(N \widetilde{N \mathcal{F}})$ is an almost transverse semi-spray and $C: N \mathcal{F} \rightarrow T M$ is a nonlinear constraint, then there is a unique vector field $\mathcal{S} \in \mathcal{X}(\widetilde{N F})$ that projects by $T \widetilde{N \mathcal{F}} \rightarrow N \widetilde{N \mathcal{F}}$ and $T \widetilde{N \mathcal{F}} \rightarrow T M$ to $S$ and $C$ respectively.

As a historically representative nonlinear example is the nonlinear Appell constraint, defined as follows. Take the foliation of $R_{0}^{3}=R^{3} \backslash\{\overline{0}\}$ generated by $\frac{\partial}{\partial z}$. Denote $x=x^{\overline{1}}, y=x^{\overline{2}}$ and $z=x^{1}$ and consider the nonlinear constraint given by the implicit equation

$$
\alpha^{2}\left(\left(y^{\overline{1}}\right)^{2}+\left(y^{\overline{2}}\right)^{2}\right)-\left(y^{1}\right)^{2}=0, \alpha \neq 0 .
$$

We can consider $y^{1}=C^{1}\left(y^{\overline{1}}, y^{\overline{2}}\right)=\alpha \sqrt{\left(y^{\overline{1}}\right)^{2}+\left(y^{\overline{2}}\right)^{2}}$

Formula for the nonlinear constraints is

$$
X^{1} \frac{\partial}{\partial x^{1}}+X^{\overline{1}} \frac{\partial}{\partial x^{\overline{1}}}+X^{\overline{2}} \frac{\partial}{\partial x^{\overline{2}}} \stackrel{C^{\prime}}{\rightarrow}\left(X^{1}+\alpha \frac{X^{\overline{1}} y^{\overline{1}}+X^{\overline{2}} y^{\overline{2}}}{\sqrt{\left(y^{\overline{1}}\right)^{2}+\left(y^{\overline{2}}\right)^{2}}}\right) \frac{\partial}{\partial x^{1}} .
$$

Let us consider $N^{T} \mathcal{F}=N \mathcal{F} \times R$ or $N^{T} \mathcal{F}=N \mathcal{F} \times S^{1}$ and the foliation $\overline{\mathcal{F}}^{T}$ on $N^{T} \mathcal{F}$ is induced by the foliation $\overline{\mathcal{F}}=\mathcal{F}_{N \mathcal{F}}$ on $N \mathcal{F}$, such that the canonical projection $N^{T} \mathcal{F} \rightarrow N \mathcal{F}$ is a diffeomorphism of leaves, thus the new parameter is transverse. A time dependent nonlinear constraint on $M$ is a map $C: N^{T} \mathcal{F} \rightarrow T M$, viewed also as a section $C \in \Gamma\left(\pi_{N^{T} \mathcal{F}}^{*} T M\right)$, such that $\tilde{J}(C)=\Gamma_{0}$. Using local coordinates,

$$
\left(x^{u}, x^{\bar{u}}, y^{\bar{u}}, t\right) \stackrel{C}{\rightarrow}\left(C^{u}\left(x^{v}, x^{\bar{v}}, y^{\bar{v}}, t\right), y^{\bar{u}}\right), C=C^{u} \frac{\partial}{\partial x^{u}}+y^{\bar{u}} \frac{\partial}{\partial x^{\bar{u}}} .
$$

There is an exact sequence, induced by (9):

$$
0 \rightarrow \pi_{N^{T} \mathcal{F}}^{*} T \mathcal{F} \stackrel{I_{0}^{\prime \prime}}{\rightarrow} \pi_{N^{T} \mathcal{F}}^{*} T M \stackrel{\Pi_{0}^{\prime \prime}}{\rightarrow} \pi_{N^{T} \mathcal{F}}^{*} N \mathcal{F} \rightarrow 0 .
$$

As in the time independent case, a time dependent nonlinear constraint on $M$ gives also rise to a left splitting $C^{\prime \prime}$ or, equivalently, a right splitting $D^{\prime \prime}$ of the exact sequence (14). Analogous decomposition as (10) can be obtained. 
Proposition 3.4 [20, Proposition 4]If $C: N \mathcal{F} \rightarrow T M$ is a nonlinear constraint and $S \in$ $X(N \mathcal{F})$ is a $C$-semispray, then the local formula

$$
-\left[\left[\frac{\partial}{\partial y^{\bar{u}}}, C_{V}\right], C_{V}\right]+S^{\bar{v}} \frac{\partial^{2} C^{u}}{\partial y^{\bar{u}} \partial y^{\bar{v}}} \frac{\partial}{\partial x^{u}}=R_{\bar{u}}^{u} \frac{\partial}{\partial x^{u}},
$$

gives a global tensor $R \in L\left(V N \mathcal{F}, T \mathcal{F}_{N \mathcal{F}}\right), R=R_{\bar{u}}^{u}\left(x^{v}, x^{\bar{v}}, y^{\bar{v}}\right) \frac{\partial}{\partial x^{u}} \otimes d x^{\bar{u}}$.

In local coordinates:

$$
-\left[C_{V},\left[C_{V}, \frac{\partial}{\partial y^{\bar{u}}}\right]\right]=\left(C^{v} \frac{\partial^{2} C^{u}}{\partial x^{v} \partial y^{\bar{u}}}+y^{\bar{v}} \frac{\partial^{2} C^{u}}{\partial x^{\bar{v}} \partial y^{\bar{u}}}-\frac{\partial C^{u}}{\partial x^{\bar{u}}}-\frac{\partial C^{v}}{\partial y^{\bar{u}}} \frac{\partial C^{u}}{\partial x^{v}}\right) \frac{\partial}{\partial x^{u}} .
$$

The nonlinear constraints effect on Lagrange equations, as for linear and affine constraints in Bloch monograph has the form:

$$
\begin{aligned}
\left(\frac{d}{d t} \frac{\partial L}{\partial y^{u}}-\frac{\partial L}{\partial x^{u}}\right) \delta x^{u}+\left(\frac{d}{d t} \frac{\partial L}{\partial y^{\bar{u}}}-\frac{\partial L}{\partial x^{\bar{u}}}\right) \delta x^{\bar{u}} & =0, \\
\delta x^{u}+C_{\bar{u}}^{u} \delta x^{\bar{u}} & =0,
\end{aligned}
$$

where $C_{\bar{u}}^{u}=\frac{\partial C^{u}}{\partial y^{\bar{u}}}$. One obtain the induced constrained Lagrange equations:

$$
\left(\frac{d}{d t} \frac{\partial L}{\partial y^{\bar{u}}}-\frac{\partial L}{\partial x^{\bar{u}}}\right)-C_{\bar{u}}^{u}\left(\frac{d}{d t} \frac{\partial L}{\partial y^{u}}-\frac{\partial L}{\partial x^{u}}\right)=0
$$

As we see below for implicit nonlinear constraints, these equations are concordant to Chetaev conditions.

A nonlinear constraint $C \in \Gamma\left(\pi_{N \mathcal{F}}^{*} T M\right)$ can be viewed as a map $C: N \mathcal{F} \rightarrow T M$, thus any lagrangian $L: T M \rightarrow R$ induces by composition $N \mathcal{F} \stackrel{C}{\rightarrow} T M \stackrel{L}{\rightarrow} R$ a new lagrangian $L_{c}=L \circ C$ on $N \mathcal{F}$, called the constrained lagrangian:

$$
L_{c}\left(x^{u}, x^{\bar{u}}, y^{\bar{u}}\right)=L\left(x^{u}, x^{\bar{u}}, C^{u}, y^{\bar{u}}\right) .
$$

Proposition 3.5 [20, Proposition 6]If the lagrangian L is C-regular, then the integral curves, solutions of equations of motion of the extended nonholonomic system, are the integral curves of a $C$-semispray $\mathcal{S}$.

Let us denote by $\mathcal{L}$ the Legendre map of $L$. The statement below contains the main result of the paper. It gives all the equations of motion in the same form as in [6], all in presence of constraints adapted to a regular foliation.

Theorem 3.1 [20, Theorem 4.1]Let $L: T M \rightarrow R$ be a lagrangian, $C: N \mathcal{F} \rightarrow T M$ be a nonlinear constraint and $L_{c}=L \circ C$ be the constrained lagrangian. If one of the following conditions holds:

1. L is C-regular, or

2. $C$ is a linear constraint, or

3. $C$ is an affine constraint 
then the constrained Lagrange equations (17) have the form

$$
\delta L_{c}=\langle R, \mathcal{L}\rangle,
$$

or, using local coordinates,

$$
\frac{d}{d t} \frac{\partial L_{c}}{\partial y^{\bar{u}}}-\frac{\partial L_{c}}{\partial x^{\bar{u}}}+C_{\bar{u}}^{u} \frac{\partial L_{c}}{\partial x^{u}}=\frac{\partial L}{\partial y^{u}} R_{\bar{u}}^{u}
$$

where, in the first case, $R$ is the $S$-curvature of $C$ and $S$ is the almost transverse semi-spray canonically associated, and, in the last two cases, $R$ is the curvature of $C$.

\section{Cotangent / Hamiltonian form}

Let us suppose that the induced lagrangian $L_{d}$ is regular and denote by $h^{\bar{u} \bar{v}}$ its hessian. The inverse of its Legendre map has the form $\left(x^{v}, x^{\bar{v}}, p_{\bar{v}}\right) \longrightarrow\left(x^{v}, x^{\bar{v}}, y^{\bar{v}}=h^{\bar{v}}\left(x^{v}, x^{\bar{v}}, p_{\bar{v}}\right)\right)$.

We have

$$
\begin{aligned}
& \frac{\partial h^{\bar{w}}}{\partial p_{\bar{v}}}=h^{\bar{v} \bar{w}}, \\
& \frac{\partial h^{\bar{w}}}{\partial x^{v}}=-\left(\frac{\partial L}{\partial x^{v} \partial y^{\bar{u}}}+\frac{\partial^{2} L}{\partial y^{w} \partial y^{\bar{u}}} \frac{\partial C^{w}}{\partial x^{v}}\right) h^{\bar{u} \bar{w}}=-\tilde{h}_{v \bar{u}} h^{\bar{u} \bar{\omega}}, \\
& \frac{\partial h^{\bar{w}}}{\partial x^{\bar{v}}}=-\left(\frac{\partial L}{\partial x^{\bar{v}} \partial y^{\bar{u}}}+\frac{\partial^{2} L}{\partial y^{w} \partial y^{\bar{u}}} \frac{\partial C^{w}}{\partial x^{\bar{v}}}\right) h^{\bar{u} \bar{w}}=-\tilde{h}_{\bar{v} \bar{u}} h^{\bar{u} \bar{w}} .
\end{aligned}
$$

Then equation (17) becomes

$$
\begin{aligned}
& \frac{d p_{\bar{w}}}{d t}\left(h_{\bar{u} \bar{v}}+C_{\bar{u}}^{u} h_{u \bar{v}}\right) h^{\bar{v} \bar{w}}-\frac{\partial L}{\partial x^{\bar{u}}}+C_{\bar{u}}^{u}\left(\left(h_{v u}-\tilde{h}_{v \bar{w}} h^{\bar{w} \bar{v}} h_{u \bar{v}}\right) C^{v}\right. \\
& \left.+\left(h_{\bar{v} u}-\tilde{h}_{\bar{v} \bar{u}} h^{\bar{u} \bar{w}} h_{u \bar{w}}\right) h^{\bar{v}}+\frac{\partial^{2} L}{\partial y^{w} \partial y^{u}} \frac{\partial C^{w}}{\partial t}-\frac{\partial L}{\partial x^{u}}\right)=0,
\end{aligned}
$$

since $\frac{d x^{v}}{d t}=C^{v}\left(x^{v}, x^{\bar{v}}, h^{\bar{v}}\right), \frac{d x^{\bar{v}}}{d t}=h^{\bar{v}}\left(x^{v}, x^{\bar{v}}, p_{\bar{v}}\right)$.

But $h_{\bar{u} \bar{v}}=\frac{\partial}{\partial y^{\bar{u}}} \frac{\partial L}{\partial y^{\bar{v}}}=\frac{\partial^{2} L}{\partial y^{\bar{u}} \partial y^{\bar{v}}}+C_{\bar{u}}^{u} \frac{\partial^{2} L}{\partial y^{u} \partial y^{\bar{v}}}$, thus $h_{\bar{u} \bar{v}}+C_{\bar{u}}^{u} h_{u \bar{v}}=\frac{\partial^{2} L}{\partial y^{\bar{u}} \partial y^{\bar{v}}}+C_{\bar{u}}^{u} \frac{\partial^{2} L}{\partial y^{u} \partial y^{\bar{v}}}+$ $C_{\bar{u}}^{u} \frac{\partial^{2} L}{\partial y^{\bar{v}} \partial y^{u}}+\frac{\partial^{2} L}{\partial y^{w} \partial y^{u}} C_{\bar{v}}^{w} C_{\bar{u}}^{u}$ is the restriction of the Hessian of $L$ to the constrained manifold.

Theorem 4.1 Let us denote $H_{\bar{u}}^{\bar{w}}=\left(h_{\bar{u} \bar{v}}+C_{\bar{u}}^{u} h_{u \bar{v}}\right) h^{\bar{v} \bar{w}}$. If there exists $\left(H_{\bar{u}}^{\bar{w}}\right)^{-1}=\left(\tilde{H}_{\bar{v}}^{\bar{u}}\right)$, then the constrained Lagrange equations (17) have the cotangent form

$$
\begin{aligned}
& \frac{d p_{\bar{w}}}{d t}+H_{\bar{w}}^{\bar{u}}\left(\frac{\partial H}{\partial x^{\bar{u}}}+C_{\bar{u}}^{u}\left(\left(h_{v u}-\tilde{h}_{v \bar{t}} h^{\overline{\bar{v}}} h_{u \bar{v}}\right) C^{v}\right.\right. \\
& \left.\left.+\left(h_{\bar{v} u}-\tilde{h}_{\bar{v} \bar{u}} h^{\bar{u} \bar{t}} h_{u \bar{t}}\right) h^{\bar{v}}+g_{w u} \frac{\partial C^{w}}{\partial t}+\frac{\partial H}{\partial x^{u}}\right)\right)=0,
\end{aligned}
$$

where $H\left(x^{u}, x^{\bar{u}}, p_{u}, p_{\bar{u}}\right)=p_{u} C^{u}+p_{\bar{u}} h^{\bar{u}}\left(x^{u}, x^{\bar{u}}, p_{\bar{u}}\right)-L\left(x^{u}, x^{\bar{u}}, C^{u}, h^{\bar{u}}\right)$ and $g_{u v}=\frac{\partial^{2} L}{\partial y^{w} \partial y^{u}}$ correspond to the inverse of the Legendre map. 


\section{An example: a riemannian flow}

As an example, one can consider a riemannian flow on a manifold $M$, i.e. a one-dimensional riemannian foliation. The Lagrangian is not time dependent, but the constraints are. Thus there are given on $M$ :

- a one-dimensional distribution (given, for example, by a non-vanishing vector field $X_{0}$ ) and - a bundle-like metric $g$.

Using local coordinates $\left(x^{1}, x^{\bar{u}}\right)$ on $M$, adapted to the foliation, the vector field $X_{0}$ and the quadratic lagrangian of the metric $g$ have the local forms

$$
\begin{aligned}
X_{0} & =X^{0}\left(x^{0}, x^{\bar{u}}\right) \frac{\partial}{\partial x^{0}}, \\
L\left(x^{0}, x^{\bar{u}}, y^{0}, y^{\bar{u}}\right) & =\frac{1}{2} g_{0}\left(x^{0}, x^{\bar{u}}\right)^{2}\left(y^{0}\right)^{2}+\frac{1}{2} g_{\bar{u} \bar{v}}\left(x^{\bar{u}}\right) y^{\bar{u}} y^{\bar{v}},
\end{aligned}
$$

where $Y^{\bar{u}}=y^{\bar{u}}+y^{0} \tilde{g}_{0}^{\bar{u}}\left(x^{0}, x^{\bar{v}}\right)$. We notice that $g_{0}$ can be taken 1 only in the case when the orthogonal distribution to the riemannian flow is integrable.

A quadratic time-dependent constraint has the form

$$
L\left(x^{1}, x^{\bar{u}}, y^{1}, y^{\bar{u}}\right)=\frac{1}{2} \varphi(t)
$$

This applies in the particular case of a decelerated motion of a free particle, studied initially in [12] and then in [22], where the explicit form of the trajectories are obtained. In this case $\varphi(t)=\frac{1}{t}$, when $\Phi=2 \sqrt{t}$.

We have

$$
\frac{d y^{\bar{v}}}{d t}=\frac{y^{\bar{v}} \varphi^{\prime}}{2 \varphi} .
$$

It follows that $y^{\bar{v}}=c_{\bar{v}} \sqrt{\varphi}$. Since $y^{\bar{v}}=\frac{d x^{\bar{v}}}{d t}$, it follows that the trajectories have the local form

$$
x^{\bar{v}}=c_{\bar{v}} \Phi(t)+d_{\bar{v}},
$$

where $c_{\bar{v}}$ and $d_{\bar{v}}$ are constants and $\Phi(t) \in \int \sqrt{\varphi(t)} d t$, i.e. a primitive of $\sqrt{\varphi(t)}$.

In the hamiltonian form, we obtain

$$
\frac{d p_{\bar{v}}}{d t}=\frac{\varphi^{\prime}}{2 \varphi} p_{\bar{v}}
$$

thus in accordance with formula (20).

\section{References}

[1] L.M. Bates, Personal communication.

[2] L.M. Bates, and J. M. Nester, On D' Alembert's principle, Communications in Mathematics 19(1), 57-72 (2011).

[3] S. Benenti, Geometrical aspects of the dynamics of non-holonomic systems, Rend. Sem. Mat. Univ. Pol. Torino 54, 203-212 (1996). 
[4] A. Bejancu, Nonholonomic mechanical systems and Kaluza-Klein theory, Journal of Nonlinear Science 22, 213-233 (2012).

[5] A. Bejancu A., H.R. Farran, Foliations and Geometric Structures (Springer, Series: Mathematics and Its Applications Vol. 580, 2006) 580 pp.

[6] A. M. Bloch, Nonholonomic Mechanics and Control (Vol. 24, Springer, 2003) 503 pp.

[7] I. Bucataru and R. Miron, Finsler-Lagrange geometry: Applications to dynamical systems (Editura Academiei Romane, Bucuresti, 2007) 264 pp.

[8] M. R. Flannery, The enigma of nonholonomic constraints, American Journal of Physics 73(3), 265-272 (2005).

[9] Y.-X. Guo, J. Li-Yan and Y. Ying, Symmetries of mechanical systems with nonlinear nonholonomic constraints, Chinese Physics 10, p. 181 (2001).

[10] K. Grabowska and J. Grabowski, Variational calculus with constraints on general algebroids, Journal of Physics A: Mathematical and Theoretical 41, 175-204 (2008).

[11] M. H. Kobayashi, W. M. Oliva, A note on the conservation of energy and volume in the setting of nonholonomic mechanical systems, Qualitative Theory of Dynamical Systems 4, 383-411 (2004).

[12] O. Krupková, Mechanical systems with nonholonomic constraints, Journal of Mathematical Physics 38, 5098-5126 (1997).

[13] S. Lang, Differential and Riemannian Manifolds (Springer Verlag, New York, 1995) $378 \mathrm{pp}$.

[14] M. de León M., A historical review on nonholonomic mechanics, Revista de la Real Academia de Ciencias Exactas, Fisicas Y Naturales (Serie A: Matematicas) 105 (2011).

[15] M. de León, D. Martín de Diego and M. Vaquero, A Hamilton-Jacobi theory on Poisson manifolds, Journal of Geometric Mechanics 6, 121-140 (2014).

[16] S.-M. Li and J. Berakdar, A generalization of the Chetaev condition for nonlinear nonholonomic constraints: The velocity-determined virtual displacement approach, Reports on Mathematical Physics 63, 179-189 (2009).

[17] C. M. Marle, Kinematic and geometric constraints, servomechanisms and control of mechanical systems, Rend. Sem. Mat. Univ. Pol. Torino 54, 353-364 (1996).

[18] C. M. Marle, Various approaches to conservative and nonconservative nonholonomic systems, Reports on Mathematical Physics 42, 211-229 (1998).

[19] P. Molino, Riemannian Foliations (Birkhäuser, Progr. Math. 73, 1988) 356 pp.

[20] P. Popescu, C. Ida, Nonlinear constraints in nonholonomic mechanics, Journal of Geometric Mechanics 6(4), 527-547 (2014).

[21] P. Popescu and M. Popescu, Lagrangians adapted to submersions and foliations, Differential Geom. Appl. 27, 171-178 (2009).

[22] M. Swaczyna, Several examples of nonholonomic mechanical systems, Communications in Mathematics 19(1), 27-56 (2011). 